\title{
Routing in Vehicular Networks: Feasibility, Modeling, and Security
}

\author{
Ioannis Broustis and Michalis Faloutsos \\ Department of Computer Science and Engineering, University of California - Riverside, Riverside, CA 92521, USA
}

Correspondence should be addressed to Broustis Ioannis, broustis@cs.ucr.edu

Received 11 July 2007; Accepted 19 March 2008

Recommended by Jianping Pan

Vehicular networks are sets of surface transportation systems that have the ability to communicate with each other. There are several possible network architectures to organize their in-vehicle computing systems. Potential schemes may include vehicle-tovehicle ad hoc networks, wired backbone with wireless last hops, or hybrid architectures using vehicle-to-vehicle communications to augment roadside communication infrastructures. Some special properties of these networks, such as high mobility, network partitioning, and constrained topology, differentiate them from other types of wireless networks. We provide an in-depth discussion on the important studies related to architectural design and routing for such networks. Moreover, we discuss the major security concerns appearing in vehicular networks.

Copyright (C) 2008 I. Broustis and M. Faloutsos. This is an open access article distributed under the Creative Commons Attribution License, which permits unrestricted use, distribution, and reproduction in any medium, provided the original work is properly cited.

\section{Introduction}

The widespread proliferation of computing systems, during the last decade, has enabled the setup and deployment of vehicular networks in all kinds of environments. The FCC has allocated $75 \mathrm{MHz}$ of spectrum at $5.9 \mathrm{GHz}$ for shortrange communications between vehicles and from vehicles to roadside facilities. Such networks offer the potential for fast and accurate driving information (e.g., traffic, accidents, and emissions) that would otherwise be more difficult to disseminate. Hence, new ways to improve and optimize the transportation system are enabled. Also a variety of commercial applications can be easily supported. Vehicular networks can be used to facilitate the service customization to the needs of individual nodes. Possible applications for such networks can be generally classified as safety and nonsafety applications. Safety applications include accident avoidance and cooperative driving [1]. Nonsafety applications include traffic information [2], toll service, Internet access [3], cooperative entertainment, and so on.

Vehicular networks consist of nodes-vehicles equipped with wireless communication devices, GPS, digital maps, and optional sensors for reporting the vehicle condition. Vehicles exchange information with other vehicles as well as with access points (base stations) within their radio range. Ad hoc or infrastructure wireless networks are used to propagate information. However, the data propagation requires innovative routing algorithms. This is because, as we explain later, vehicular networks have unique characteristics that differentiate them from common wireless networks. As a consequence, routing is a challenging task due to the high dynamics of such a network.

In this article, we investigate the following. (1) The extent to which the vehicular network characteristics can determine the performance of routing. (2) How can current wireless technologies (e.g., WiFi, UWB, WiMax, and cellular) support vehicular networks? (3) Most of the important related studies on vehicular network architectures and routing. We do not focus specifically on one type of architecture; in contrast we describe and comment on most currently famous suggested schemes. (4) Finally, how feasible is to maintain a level of security in such networks?

The remainder of this article is organized as follows. In Section 2, we discuss the unique characteristics of vehicular networks, and how these demand innovative routing protocols. In Section 3, we present related studies on vehicular network routing. We also add our comments about the validity and the importance of them. Some routing and topological 
security concerns are mentioned in Section 4. Specifically, we discuss how can the vehicular network properties affect the impact of malicious attacks, as well as some general mitigation schemes. Finally, Section 5 concludes this paper.

\section{Background}

In this section, we first describe the unique properties of vehicular networks. Next, we discuss the applicability of current wireless technologies on vehicular networks. Finally, we discuss potential applications for such networks.

\subsection{Vehicular Network Properties}

As we mentioned earlier, vehicular networks have specific characteristics. These properties affect the decisions that designers have to take when building architectures for such networks. This is because some of their characteristics prohibit the use of current routing protocols that are applicable to regular wireless networks. Here we discuss these uniquenesses.

\section{Geographically Constrained Topology}

Roads limit the network topology to actually one dimension: the road direction. Except for crossroads or overlay bridges, roads are generally located far apart. Even in urban areas, where they are located close to each other, there exist obstacles, such as buildings and advertisement walls, which prevent wireless signals from traveling between roads. This implies that nodes-vehicles can be considered as points of the same line; a road can be approximated as a straight line or a small-angled curve. This observation is quite important, because it affects the wireless technologies that can be considered. For example, since the packet relays are almost all in the same one-directional deployment region, the use of directional antennas could be of great advantage.

\section{Partitioning and Large Scale}

In vehicular networks, the probability of end-to-end connectivity decreases with distance [4]; this is true for onedimensional network topologies. In contrast, connectivity is often explicitly assumed in research for traditional ad hoc networks, sometimes even for the evaluation of routing protocols. In addition, vehicular networks can extend in large areas as far as there is road available. This artifact together with the one-dimensional deployment increases the above probability.

\section{Predictable Mobility}

Because vehicle mobility depends on the deployment scenario, the movement direction is predictable to some extent. In highways, vehicles often move at high speeds, while in urban areas they are slow. In addition, mobility is restricted by the road directions as well as by traffic regulations. Assuming that these regulations are obeyed, there are lower and upper speed bounds, and restriction signs that obligate drivers to move on specific roads and directions. Hence, mobility models can now include some level of predictability in movement patterns. Car manufacturing companies have already implemented such models for testing mechanical parts.

\section{Power Consumption}

In traditional wireless networks, nodes are power limited and their life depends on their batteries (this is especially the case for ad hoc networks). Vehicles however can provide continuous power to their computing and communication devices. As a result, routing protocols do not have to account for methodologies that try to prolong the battery life. Older network protocols include mechanisms such as battery-life reports for energy-efficient path selection, sleepawake intervals, as well as advanced network/MAC crosslayer coordination algorithms. These schemes cannot offer any additional advantages to vehicular networks.

\section{Node Reliability}

Vehicles may join and leave the network at any time and much more frequently than in other wireless networks. The arrival/departure rate depends on their speed, the environment, as well as on the drivers' needs to be connected to the network. Especially for ad hoc deployments, the network cannot easily depend on a single vehicle for packet forwarding. This is because the duration of the vehicle's cooperation depends on its destination. Also, apart from vehicles failing in unpredictable ways, security issues come into play. We discuss these issues later in this article.

\subsection{Potentially Applicable Wireless Technologies}

There are three potential wireless technologies under discussion that can be adopted: wireless metropolitan area networks (WMANs), wireless local area networks (WLANs/WiFi), and wireless personal area networks (WPANs), together with their ad hoc mode of operation.

\section{WMANs}

A WMAN (wireless metropolitan area network) can interconnect distant locations. Two kinds of WMANs exist: back haul and last mile. Back haul is for enterprise networks, cellular base station communications, and Wi-Fi hotspots. A private WMAN broadband system is a quite chip solution and it is 10 times faster than a DSL or T1 wireline connection. Thus, it is affordable for companies that do not wish to pay double the price for a fiber 10-Mbps link to their ISPs. Last-mile setups can establish wireless as an alternative to residential broadband modems. In a typical cell radius deployment of three to ten kilometers, last mile systems can be expected to deliver capacity of up to $40 \mathrm{Mbps}$ per channel. This is enough bandwidth to simultaneously support hundreds of businesses with T1 speed connectivity and thousands of residences with DSL speed connectivity. WMAN connections can be PTP (point-to-point) or PMP 
(point-to-multipoint). Both omnidirectional and directional antennas can be used, as well as dynamically alternated radio channels and antenna polarization. PMP setups, where a central point serves multiple remote sites, are preferable when the density of links is high. PMP systems typically use a polling protocol to support high-density applications. One of the most interesting recent developments is the standardization of WMANs in the form of IEEE 802.16. Finally, the WMAN category also includes the GSM/GPRS cellular infrastructure networks.

The WMAN type of technology could be employed in infrastructure-based vehicular networks alone, or in coordination with WLANs or WPANs (and their ad hoc multihop types) as last-hops. WiMax promises to bring wireless high-speed connections to entire metropolitan areas. It is currently supported by 140 companies. WiMax has a reach of 1 to 10 miles, offering a way to bring the Internet to entire communities. Mobile network deployments are expected to provide up to $15 \mathrm{Mbps}$ of capacity within a typical cell radius deployment of up to 3 kilometers. This is an obviously high-potential solution for vehicular networks, even for distant highway environments.

When collaborating with WiFi/WPANs, the WMAN may provide the permanent connectivity. The PAN/WiFi portion could be added from the base stations to the vehicles, as well as among vehicles themselves (V2V or Vehicle-To-Vehicle multihop communications) to offer high bandwidth with low cost. Also, a potential protocol could support the direct connectivity of a vehicle with the WMAN, either when there is lack of a WLAN base station in that area, or when the number of hops to the base station exceeds some threshold. An alternative could also be to maintain permanent direct links from vehicles to cellular base stations, without the direct communication among vehicles. However, from the cellular network perspective, this will probably result in a relatively low throughput. Currently, the GSM/GPRS technology ideally offers at most $100 \mathrm{Kbps}$ of bandwidth. Also 3G systems can reach $384 \mathrm{Kbps}$. Future cellular PHY technologies may provide higher throughputs, allowing more data rate-demanding applications to be supported.

\section{WLANs/WiFi}

WiFi is another possibility for vehicular networks. An IEEE 802.11 transmitter has a 250-meter omnidirectional coverage range, which is potentially enough to maintain a level of multihop connectivity in both highway and urban regions. In addition, extended-vicinity antennas (umbrellas) could be employed in base stations, for covering larger distances. A lot of research has been done for the popular IEEE 802.11 wireless protocol, mostly for the MAC (CSMA/CA) and network layers. However, this research cannot be taken "off the shelf" for use in vehicular networks. This is because of the unique properties that we described above.

\section{WPANs}

Wireless personal area networks are used for short-range wireless communications. Two of the most popular tech- nologies, Bluetooth and ultrawide band (UWB), belong to this category. While the former offers a low data rate (up to $10 \mathrm{Mbps}$ for Bluetooth v2.0), the latter promises very high data rates, up to $500 \mathrm{Mbps}$, over short distances. Even though there has been a lot of work done for the PHY layer or UWB, concerning modulation and channelization, only a few studies exist for upper layers. Especially for UWB ad hoc networks, MAC and network layer protocols are still under consideration. Even though the data rates offered by UWB are tempting, the short transmission range (maximum 10$20 \mathrm{~m}$ ) restricts the applicability of this technology to only very dense urban-area vehicular networks.

\section{Millimeter-wave Communications}

The $60 \mathrm{GHz}$ band is located in the millimeter-wave portion of the RF spectrum. This part of the spectrum, although very promising, is largely unexplored with only a few companies producing FCC-approved wireless products. The major advantages of this technology are the following [5]. (1) It can offer extremely high data rates (due to the huge available bandwidth) comparable to fiber-optics performance, over hundred of meters. (2) Very low levels of interference, as well as high levels of security, mainly due to oxygen absorption and narrow antenna beams. (3) Potentially high level of frequency reuse. Communications over the $60 \mathrm{GHz}$ band are promising for applications over vehicular networks. However, this technology is still quite unknown and unexplored, with only a few research achievements in its background.

In summary, we believe that the most appropriate wireless technology for vehicular networks, to-date, is the WMAN technology alone, or WMAN in cooperation with WiFi and sometimes with WPANs. The high mobility as well as the network partitioning and scalability demand the employment of either infrastructure-based wireless infrastructures or scalable adhoc solutions, such as hierarchical clustering structures, and so on. Note also that the millimeter-wave technology can be largely applicable as long as significant research efforts are expended towards this solution.

\subsection{Applications}

Applications running on top of vehicular networks can be categorized as safety and nonsafety applications: drivervehicle safety, infotainment, and mobile internet services for passengers. In addition to low cost and robust wireless communication devices, vehicles can also be equipped with storage, processing, and sensing equipment. Vehicles can be used as store-and-forward mobile routers, on-demand and dynamic grid computing engines, as well as distributed mobile sensor networks.

There are a number of projects, completed or under development, targeting to improve roadway conditions. The Fleetnet project [3], funded by the German Federal Ministry for Education and Research, focuses on mobile ad hoc radio networks. Fleenet applications include emergency braking notification and traffic data distribution. The VMesh/VGrid project [6] has two directions. In VMesh, 
vehicles dynamically form a mobile transit network to gather and dessiminate information. For example, data may be relayed between different clusters of static nodes that are otherwise disconnected. VGrid targets to evolve intelligent transportation system (ITS) from a centralized to a distributed approach, in which vehicles can cooperatively solve traffic-flow control problems. Furthermore, one of the most intelligent transportation systems exists in Singapore. It includes real-time surveillance of road speeds, road pricing, advanced traffic signal control, and an advanced mass transit system. Near-future "smart" vehicular computational devices of various types will be able to communicate with each other and utilize their diverse resources: wireless networks, embedded processors and sensors, databases, satellites, and so on. This implies the need for innovative communication protocols, specialized to adopt the unique properties of vehicular networks and the availability of their resources.

An interesting information architecture toolkit is discussed in [7]. It includes (1) wireless networking capabilities, (2) traffic prediction algorithms, (3) vehicle and trajectory recognition based on fusing heterogeneous data, (4) cost models (fairness, robustness, privacy, computational efficiency), and (5) real-time maintenance, prediction and generation of spatiotemporal information. The kit can be used in a variety of applications: planning multimodal routes, exchange of real-time traffic information, autonomous unmanned vehicle driving and, of course, multivehicle cooperation (MVEC). For this latter application, vehicles are assumed to be equipped with GPS receivers, computational devices, and wireless communication systems. Vehicles will be able to process queries, such as "what is the average vehicle speed 2 miles ahead?". Processing such queries demands multihop links and mobile-database utilization.

\section{Related Studies on Vehicular Routing}

There has been a lot of interest to exploit the potentials of vehicular networks. However, only a few studies propose complete routing solutions and architectures. In this section, we present the most important of these studies. We may have various categorizations for them. One could be to separate them according to the type of architecture that they import: ad hoc or infrastructure or hybrid. Another categorization could involve the deployment region: highway or urban regions. Below we describe and discuss the most important ones.

Most studies in vehicular routing focus either on comparing current routing solutions for traditional wireless networks, or describing issues that must be taken into account, when building appropriate models. In [8] Füßler et al. examine the applicability of existing ad hoc routing protocols to VANETs. Specifically, they compare the famous Dynamic Source Routing (DSR) and the Greedy Perimeter Stateless Routing (GPSR) protocols. They conclude that when communication sessions are comprised or more than 2 or 3 hops, position-based ad hoc routing is preferable over reactive nonposition-based approaches. The advantages have to do with both the successfully delivered packets and the control overhead. In addition, the authors argue that the random waypoint model is rather inappropriate to accurately reproduce vehicle movement. Alternatively, they make use of the well-validated FARSI simulator, adopted by many car companies to generate traffic simulation scenarios. For their simulations, they assume the deployment of the IEEE 802.11 protocol. They show that current position-based schemes provide high data rates, even over many hops. Moreover, the overhead is small and does not impact on scalability. The reason is that position-based routing does not store routes and instead performs forwarding on the fly. An improvement to DSR could involve the movement of individual vehicles in the routing decision. Thus, preference to routes over vehicles moving in the same direction can be given. As a result, topological changes would be infrequent. Finally, for position-based schemes, they generally propose caching and prediction of a node's location, based on its speed and direction.

\section{$M D D V$}

The mobility-centric data dissemination algorithm (MDDV) [9] is one of the few that provide a complete architecture for vehicular routing. It combines the ideas of opportunistic forwarding, trajectory-based forwarding, and geographical forwarding. The protocol disseminates data to intended receivers, while maintaining some design demands (e.g., high-delivery ratio, low delay, and low-memory occupancy). Even though MDDV can be applied to hybrid architectures, it is considered in VANET scenarios only.

A forwarding trajectory is a predefined path, extending from the source to the destination region. Moreover, the road network can be thought of as a directed graph, with nodes representing intersections and edges being the road segments. One approach would consider taking the shortest (road) graph distance from the source to the destination region. However, this does not imply the lowest delay, since node density often leads to fast propagation. Thus, the authors define

(1) $d(A, B)$ as the dissemination length of the road segment from node $A$ to node $B$, considering static road information;

(2) $r(A, B)$ as the road length from $\mathrm{A}$ to $\mathrm{B}$. Intuitively, when $j=0$ and $i=1$, we have $d(A, B)=r(A, B)$;

(3) $i / j$ as the number of lanes from $\mathrm{A} / \mathrm{B}$ to $\mathrm{B} / \mathrm{A}$.

For the dissemination length, the following formula is used:

$$
d(A, B)=r(A, B)\left(m-(m-1)\left(i^{p}+c j^{p}\right)\right) .
$$

The constants $p$ and $c$ take values between 0 and 1 . Constant $m$ is set to 5 .

The dissemination length is used as weight for the corresponding link in the graph. The dissemination process has two phases: the forwarding phase and the propagation phase, described below. Because no end-to-end connectivity is assumed, messages are forwarded along the forwarding trajectory through intermediate nodes; these store and forward messages opportunistically. The vehicle that holds the message and is the closest one to the destination region is 
called the message head. To increase reliability, MDDV allows a set of nodes near the message head to actively forward the message, instead of the message head alone. However, this also implies overhead increment. The design issues include the forwarding group identification, the data exchange procedure, and the decision to store/drop messages. Each node decides whether it will participate in forwarding or not, based on the traffic information in the area, as well as with some approximate knowledge of the message head location. The message head location never moves backward; a new message head location is closer to the destination than previous ones. In a nut shell, the data exchange steps are the following.

\section{(a) Forwarding Phase}

The message to be sent is assigned an owner. Usually the owner is the same as the head. Only the message owner may transmit the certain message, and the owner can be in either one of two states: active or passive. In the active state, it runs the full protocol to actively propagate the message. In the passive state, it only transmits the message if it hears an older version of it.

\section{(b) Propagation Phase}

It is initiated once the message reaches the destination region. The message now further propagates to each vehicle in the area centered at the destination, before the message time expires. In this phase, the message owner can either be in the active state or not transmitting at all. During this phase, the message is delivered to its recipient(s).

The paper provides a more detailed explanation of the algorithm, which we avoid reproducing here. It also presents the scheme in only one forwarding trajectory. However, many of them could be defined to increase robustness. Also, simulation results show the improved efficiency with regards to two simple schemes.

\section{GSR}

In [10] the Geographic Source Routing protocol is proposed. The work in [10] examines the problems appearing with baseline position-based routing in two-dimensional urban scenarios. GSR combines position-based routing with topological information. The adoption of the RLS [11] system is assumed. The source uses flooding to request the position of a node identifier. As soon as that node receives the request, it sends a position response back to the source. After discovering the location of the recipient, the source uses a digital map of the roads to calculate the set of junctions that the packet will follow. This set can be either imported to the packet header, or be derived by every forwarding node. This latter approach can be implemented on the basis of greedy forwarding. The paper discovers the source-destination route through the Dijkstra algorithm. An issue arises from the fact that the paper compares GSR with nonposition-based protocols only. There is no comparison with other positionbased schemes, such as GPSR, proposed 3 years earlier.

\section{A-STAR}

The authors in [12] present A-STAR, an anchor-based street and traffic aware routing scheme. They use information on city bus routes to identify an anchor path with high connectivity for packet delivery. The model is designed based on position-based routing, specifically to facilitate VANETs in urban areas. In such environments, vehicle density is larger in some famous (for their traffic) roads than in others. Connectivity in such roads can be higher and more stable due to regular bus passes. Also buildings constrain the signal propagation. Hence, it is more difficult to establish wireless connectivity in urban areas; the network efficiency is decreased.

A-STAR constructs a graph, based on how many bus lines go through certain roads. The number of lines determines the link weight for the certain edge of the graph. The more the routes, the less the weight. Since each vehicle may be aware of the bus route information through digital maps, an anchor route may be constructed using Dijkstra's algorithm for the least weight. Maps with preconfigured routes are called statically rated maps. In contrast, a dynamically rated one can be utilized. In such a digital map, weight assignment is performed dynamically by periodically monitoring the street traffic and updating the graph weights. Message propagation from the source to the destination follows the route produced by Dijkstra's algorithm.

The protocol includes its own local route recovery. The local recovery mechanisms adopted by other protocols, have been proven to be inefficient in urban areas because of the greedy-forwarding phase. To solve this problem, A-STAR discovers new anchor paths from the local maximum to which the packet is routed. To prevent other packets from traversing through the same region, local-maximum streets are marked as OFF. This route information is disseminated in the network, so as for these routes not to be used for anchor discovery. The protocol is simulated extensively in [12], compared to GSR and GPSR. It shows obvious network performance improvement.

\section{P2P}

A peer-to-peer approach for the support of traffic safety applications is presented in [13]. The vehicles (and potential road side access points communicate via an ad hoc peerto-peer mechanism. The exchanged data is assumed to be describing vehicular motion, road properties, and warnings or infotainment data to facilitate traffic safety. However, the scheme can also be applicable for other types of applications. Moreover, even though the paper assumes the existence of roadside servers or relays, all the network equipment is considered as part of the same vehicular network. Network nodes are called vehicular peers and they utilize adhoc connectivity. They are organized in zones, called peer spaces, according to their common interests. Each peer in a peer space maintains information about all the other peers 


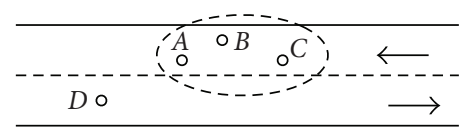

FIGURE 1: Cluster-based organization.

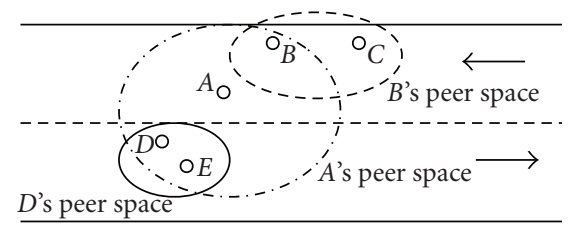

FIGURE 2: Peer-centered organization.

within the same peer space. Because the authors focus explicitly on traffic safety, they organize the peer spaces based on three issues: the communication region, the peer space composition, and specific parameters of the driving situation. They argue that there is no advantage for a peer to maintain knowledge for many others. Thus, each peer space includes at most a number of nodes; they set this to 15 peers. By this way, information overflow and high overhead are avoided. Peer space organization can be either cluster-based or peer-centered.

Vehicles decide that their safety will benefit from associating with neighbors and thus form the peer spaces. An example is depicted in Figure 1. Vehicle $A$ exchanges information with vehicles $B$ and $C$ and realizes that this data is valuable; hence vehicle A joins their network. In contrast, node $D$ considers this data useless, so it stays out of the peer space comprised by $A, B$, and $C$. When a node leaves the cluster, all remaining nodes delete all data for it. Also if a peer does not receive information about clusters in the area, it will initiate its own peer space.

In the peer-centered organization, each vehicle creates its own peer space. It analyzes data from other participants and decides which neighbor should be included in its dynamic peer space. Different peer spaces can be overlapped, as shown in Figure 2.

The major difference between the approaches is that the peer-center assumes a peer as the core of a group. The network is organized according to individual preferences. As a result, such an approach is more appropriate for urban areas. In contrast, cluster-based is preferable for highway environments.

The architecture incorporates two kinds of routing: interspace (between the peer spaces) and intraspace (within a peer space). Interspace routing is associated with traffic safety, from the perspective of accident notification to many vehicles on the road. For intraspace routing, the authors propose mediation mechanisms. All peers include in their packets the identities of the other peers that are known by senders to be registered in the same peer space. This information is stored by nodes that receive it. Highly inefficient flooding can be thus avoided.

The mediation mechanisms employed differ for the cluster-based and peer-center approaches. For the former, they can be automatic or on demand. In automatic media- tion, an individual peer is able to process and analyze data that are transmitted towards another peer in the network. It can thus determine when a peer has no data for another peer in the space. In such a case, it will retransmit the missing data. In the on-demand case, peers that are missing data for others transmit certain messages requesting the missing data. For the peer-centered organization, mediation can be automatic only.

\section{Other Schemes}

So far we discussed in some detail the most relevant related studies on vehicular network routing. Here we mention some additional work.

In [14] Saha and Johnson present a realistic model for vehicular motion, which they integrate in the famous $n s$ 2 simulator, and argue that their model is more accurate than the random way-point model in some cases of vehicular movement. The region map is represented as a graph in which vertices are the road intersections and edges are the road segments. Each node starts at a random point and moves towards another random point located on a random destination node. Dijkstra's algorithm is used to calculate the route and movement of the vehicle is constrained along this path.

Chisalita and Shahmehri in [15] propose a distributed protocol for decentralized network organization. The protocol requires the receivers to analyze the exchanged messages so as to figure out if they are the intended destinations. For this filtering, the current traffic conditions are taken into account. The protocol includes mechanisms for message acceptance/denial, local maintenance of neighborhood information, and transmission of basic safety (as well as nonsafety) messages.

In [16], Namboodiri et al. study the feasibility of mobile gateways in vehicular ad hoc networks through simulations. They use a simple mobility model, and various aspects of connectivity along with routing performance are evaluated. Simulation suggests that each vehicle should be able to connect to at least one gateway most of the time. The authors evaluate the effectiveness of the AODV routing protocol and conclude that it performs well, however they observe frequent link failures. To resolve this, they propose two simple prediction-based routing protocols to reduce those failures.

In [17] Yanlin et al. associate each vehicle with a sector, a closed area managed by several road-side units. They propose a single-hop agent advertisement and a single-phase routing scheme, which provides high packet delivery rates and low overhead.

Furthermore, Ding et al. in [18] propose SADV (staticnode assisted adaptive data dissemination), a protocol for assisting in data relaying, through the use of static nodes at road intersections. With SADV, packets are temporarily buffered in static nodes until there are vehicles within the communication range along the best delivery path to further forward the packets.

The discovery of Internet gateways by vehicles is investigated in [19]. Stationary Internet gateways are assumed 
at the roadside. Bechler et al. prove that current routing approaches and classic discovery protocols cannot address this requirement. They further propose DRIVE, a mechanism that efficiently discovers Internet gateways. This service discovery protocol employs an automated method for selecting the most suitable gateway among the available ones. It uses a fuzzy approach that considers the network properties and application classes. The location-based service discovery is also examined in [20]. Klimin et al. propose a mechanism based on geocast addressing of control messages. This hybrid approach combines request propagation reactively with a proactive method for service advertisements. The advantage from this combination is twofold. First, clients are able to initiate discovery, even when they are located outside the proactive zone of a service provider. Second, intermediate nodes may reply to service requests on the border of the provider's proactive zone. This helps saving bandwidth and accelerates the discovery procedure.

\section{Security Issues}

Generally, attacks cause anomalies to the network functionality. A lot of previous studies have investigated security vulnerabilities of routing protocols for wireless networks. These studies discuss the steps that certain attacks follow to harm the network. Such attacks can take advantage of algorithmic properties of the routing protocols. Also, there are attacks in which malicious nodes advertise fake locations to their neighbor nodes.

As for the first category, routing protocol designers need to incorporate security measures into the protocols. A designer needs to consider every aspect of his/her algorithm that could be utilized by malicious nodes. The network characteristics must also be taken into account. For example, situations in which the network topology changes dynamically are tempting to attackers for various reasons. First of all, mobility allows a modification of the routing table of the victim node simply by moving into the coverage range of it. The attacker may move away once it succeeds and without being traced. Moreover, the mobility of legitimate nodes may help attackers disperse their malicious information (epidemic spreading). Furthermore, the set of devices within the transmission range of a node keeps changing dynamically. Besides the algorithmic vulnerabilities, malicious attackers may damage the network by announcing fake node locations. Such attacks are even more difficult to mitigate.

\section{The Case of Vehicular Networks}

The unique properties of vehicular networks that we discussed earlier have an impact on attack effectiveness. First of all, attacks that target in exhausting the node battery are not applicable here. Vehicles have the ability of constantly charging their batteries. Moreover, the vehicle's power supply is more than enough to support energy-demanding computational systems. As a result, authentication processes do not have to be light-weight.

However, vehicular networks could suffer from other types of attacks. Specifically, in [4] Dousse et al. prove that the probability of end-to-end connectivity decreases with distance, for one-dimensional network topologies. This implies that it now becomes much easier for a malicious attacker to partition the network. This effect can potentially be addressed by maintaining multiple forwarding nodes for each packet. For example, in MDDV the protocol allows a group of vehicles near the message head to actively propagate the message. Hence, if we only have one or a few malicious nodes, the rest of them could potentially maintain the node reliability. However, a synchronized attack by multiple compromised vehicles would be disastrous. More than that, vehicular networks are expected to show large scalability. This, together with the unreliability of single vehicles, is ideal for applying even simple attacks.

On the other hand, even though vehicular movement can be quite fast, it is rather predictable. This does not mean that we can always know the exact direction of a vehicle; however, a probabilistic or stochastic approximation could be incorporated in previous authentication studies. Especially for location verification methods, the predicted mobility of the claimant could be easily employed from the verification algorithm. As a result, location estimation methods designed for traditional wireless networks can be adopted for vehicular networks, with minor modifications. No modifications may be required for some of them. This is because those mechanisms rely on signals transmitted either with the speed of light or with the speed of sound, or a combination of them. It is rather impossible that the average vehicle speeds will reach the speed of sound, at least in the near future, even for highway environments. Hence, the relative (to the mechanisms) vehicular speeds are not expected to affect the validity of these mechanisms. Our prediction could be supported by [9]; Wu et al. argue that the traffic in the opposite direction of the desired information flow is less helpful than the traffic in the same direction. Since the relative speeds in the same direction can be considered negligible, they will not affect the verification methods. This is because verifiers and claimant are expected to have the same approximate speed. In case the claimant travels in the opposite direction, it is less likely that it will be part of a network in its opposite direction. If this is the case however, it can be more difficult for verifiers to correctly estimate its actual location.

In [21] Zarki et al. discuss some general security and privacy issues. They present DAHNI, a simple vehicular communication infrastructure, without deeply analyzing its details. The article assumes no confidentiality for the transmitted data and that data is highly delay-sensitive. It is argued that

(1) no key distribution is usually required (no bulk data is transmitted and vehicles are not likely to stay in a cell for a long time);

(2) explicit handoffs are not required if communication is largely one-way (i.e., vehicles reporting their properties to the base station).

A potential simple security architecture for vehicular networks should at least include (1) digital signatures, (2) time-stamping and sequencing, and (3) a certification infrastructure. Even though the authors do not proceed 
to some kind of implementation/simulation, their scheme seems reasonable, while the required technology components are available nowadays.

\section{Conclusion}

In this paper, we investigated routing aspects of vehicular networks. We identified the potential wireless technologies, properties, architectures, security concerns, and previous studies. After presenting the proposed models, we commented on their efficiency and feasibility.

Vehicular networks are expected to be very attractive in the near future, facilitating numerous applications. However, to fully exploit their advantages, network designers need to take into account the unique characteristics of such networks.

\section{Acknowledgments}

The authors would like to thank the editor Jianping Pan as well as the anonymous reviewers for their constructive comments on their work.

\section{References}

[1] Q. Xu, R. Sengupta, D. Jiang, and D. Chrysler, "Design and analysis of highway safety communication protocol in $5.9 \mathrm{GHz}$ dedicated short range communication spectrum," in Proceedings of the 57th IEEE Semiannual Vehicular Technology Conference (VTC '03), vol. 4, pp. 2451-2455, Jeju, Korea, April 2003.

[2] L. Wischhof, A. Ebner, H. Rohling, M. Lott, and R. Halfmann, "Adaptive broadcast for travel and traffic information distribution based on inter-vehicle communication," in Proceedings of the IEEE Intelligent Vehicles Symposium (IV '03), pp. 6-11, Columbus, Ohio, USA, June 2003.

[3] M. Bechler, W. J. Franz, and L. Wolf, "Mobile internet access in FleetNet," in Proceedings of the 13th Fachtagung Kommunikation in Verteilten Systemen (KiVS '03), Leipzig, Germany, February 2003.

[4] O. Dousse, P. Thiran, and M. Hasler, "Connectivity in adhoc and hybrid networks," in Proceedings of the 21st Annual Joint Conference of the IEEE Computer and Communications Societies (INFOCOM '02), vol. 2, pp. 1079-1088, New York, NY, USA, June 2002.

[5] HXI, Hxi millimiter wave, http://terabeam-hxi.com.

[6] D. Ghosal, C. N. Chuah, and M. Zhang, "VGrid/VMesh: distributed sensing and computing with vehicular ad hoc networks," Tech. Rep. ECE-CE-2004-9, Computer Engineering Research Laboratory, University of California, Davis, Calif, USA, 2004.

[7] J. F. Dillenburg, P. C. Nelson, O. Wolfson, et al., "Applications of a transportation information architecture," in Proceedings of the IEEE International Conference on Networking, Sensing and Control (ICNCS '04), vol. 1, pp. 480-485, Taipei, Taiwan, March 2004.

[8] H. Füßler, M. Mauve, H. Hartenstein, M. Käsemann, and D. Vollmer, "A comparison of routing strategies for vehicular adhoc networks," in Proceedings of the ACM International Conference on Mobile Computing and Networking (MOBICOM '02), Atlanta, Ga, USA, September 2002.

[9] H. Wu, R. Fujimoto, R. Guensler, and M. Hunter, "MDDV: a mobility-centric data dissemination algorithm for vehicular networks," in Proceedings of the 1st ACM International Workshop on Vehicular Ad Hoc Networks (VANET '04), pp. 47-56, Philadelphia, Pa, USA, October 2004.

[10] C. Lochert, H. Hartenstein, J. Tian, H. Füßler, D. Hermann, and M. Mauve, "A routing strategy for vehicular ad hoc networks in city environments," in Proceedings of the IEEE Intelligent Vehicles Symposium (IV '03), pp. 156-161, Columbus, Ohio, USA, June 2003.

[11] M. Käsemann, H. Füßler, H. Hartenstein, and M. Mauve, "A reactive location service for mobile ad hoc networks," Tech. Rep. TR-02-014, University of Mannheim, Mannheim, Germany, 2002.

[12] G. Liu, B.-S. Lee, B.-C. Seet, C.-H. Foh, K.-J. Wong, and K.$\mathrm{K}$. Lee, "A routing strategy for metropolis vehicular communications," in Proceedings of the International Conference on Networking Technologies for Broadband and Mobile Networks (ICOIN '04), pp. 134-143, Busan, Korea, February 2004.

[13] I. Chisalita and N. Shahmehri, "A peer-to-peer approach to vehicular communication for the support of traffic safety applications," in Proceedings of the 5th IEEE International Conference on Intelligent Transportation Systems, pp. 336-341, Singapore, September 2002.

[14] A. K. Saha and D. B. Johnson, "Modeling mobility for vehicular ad-hoc networks," in Proceedings of the 1st ACM International Workshop on Vehicular Ad Hoc Networks (VANET '04), pp. 91-92, Philadelphia, Pa, USA, October 2004.

[15] I. Chisalita and N. Shahmehri, "A context-based vehicular communication protocol," in Proceedings of the 15th IEEE International Symposium on Personal, Indoor and Mobile Radio Communications (PIMRC '04), vol. 4, pp. 2820-2824, Barcelona, Spain, September 2004.

[16] V. Namboodiri, M. Agarwal, and L. Gao, "A study on the feasibility of mobile gateways for vehicular ad-hoc networks," in Proceedings of the 1st ACM International Workshop on Vehicular Ad Hoc Networks (VANET '04), pp. 66-75, Philadelphia, Pa, USA, October 2004.

[17] P. Yanlin, Z. Abichar, and J. M. Chang, "Roadside-aided routing (RAR) in vehicular networks," in Proceedings of the IEEE International Conference on Communications (ICC '06), vol. 8, pp. 3602-3607, Istanbul, Turkey, June 2006.

[18] Y. Ding, C. Wang, and L. Xiao, "A static-node assisted adaptive routing protocol in vehicular networks," in Proceedings of the 4th ACM International Workshop on Vehicular Ad Hoc Networks, pp. 59-68, Montreal, Quebec, Canada, September 2007.

[19] M. Bechler, L. Wolf, O. Storz, and W. J. Franz, "Efficient discovery of Internet gateways in future vehicular communication systems," in Proceedings of the 57th IEEE Semiannual Vehicular Technology Conference (VTC '03), vol. 2, pp. 965969, Jeju, Korea, April 2003.

[20] N. Klimin, W. Enkelmann, H. Karl, and A. Wolisz, "A hybrid approach for location-based service discovery in vehicular ad hoc networks," in Proceedings of the 1st International Workshop on Intelligent Transportation (WIT '04), Hamburg, Germany, March 2004.

[21] M. El Zarki, S. Mehrotra, G. Tsudik, and N. Venkatasubramanian, "Security issues in a future vehicular network", in Proceedings of the European Wireless Conference (EuroWireless '02), Florence, Italy, February 2002. 

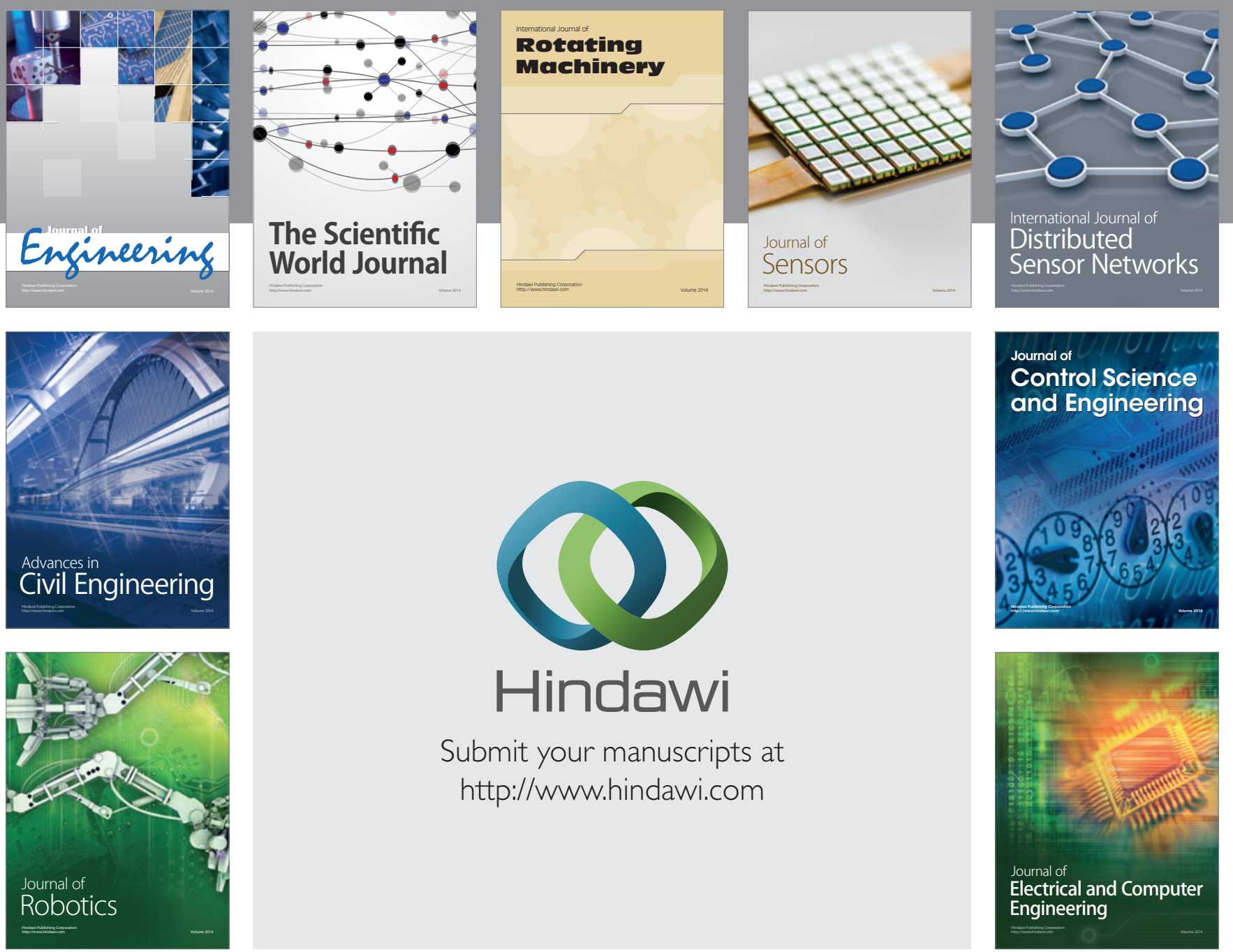

Submit your manuscripts at

http://www.hindawi.com
